\title{
The prospective analysis of functional outcome of osteosynthesis of extra-articular distal tibia fractures using minimally invasive percutaneous plate osteosynthesis technique
}

\section{Arjun Mandri, Nuthan Jagadeesh*, Sunil Nanjareddy, Vishwanath Muttagaduru Shivalingappa}

Department of Orthopedics, Vydehi Institute of Medical Sciences and Research, Bangalore, Karnataka, India

Received: 23 March 2020

Revised: 07 April 2020

Accepted: 09 April 2020

*Correspondence:

Dr. Nuthan Jagadeesh,

E-mail: nuthanjagadeesh@yahoo.com

Copyright: () the author(s), publisher and licensee Medip Academy. This is an open-access article distributed under the terms of the Creative Commons Attribution Non-Commercial License, which permits unrestricted non-commercial use, distribution, and reproduction in any medium, provided the original work is properly cited.

\begin{abstract}
Background: Extra-articular distal tibial fractures are one of the most complicated ones to treat, specially comminuted fractures. Its subcutaneous nature and lack of adequate musculature makes it more prone for soft tissue damage and bone loss. The key to handle these troubling fractures is to skilfully preserve and reconstruct the soft tissues, early mobilisation and functional use of the extremity with the maintenance of satisfactory length and alignment of the fracture.

Methods: This prospective longitudinal study involved 30 patients with extraarticular distal tibia fracture, who are admitted in Vydehi Institute of Medical Sciences and Research Centre between January 2017 to 2019. All were treated with closed reduction and internal fixation using pre-contoured anatomical distal tibia locking plate using minimally invasive percutaneous plate osteosynthesis (MIPPO) technique. Functional and radiological outcome along with associated complications are evaluated in serial follow up.

Results: 30 patients with minimal follow up criteria of 6 months was evaluated with AOFAS score for functional outcome. At the end of 1 year, 21 cases (70\%) of the cases had excellent results, 5 cases (16.7\%) had good results, 3 cases had fair results and only 1 case had poor result.

Conclusions: With the use of pre-contoured anatomical locking plates used, MIPPO technique is effective in extra articular distal tibia fractures where it not only helps in decreasing operating time but also in achieving excellent functional outcome with radiological union the fractures by preserving osseous vascularity and minimal soft tissue insult due to surgery.
\end{abstract}

Keywords: Distal tibia fractures, MIPPO technique, AOFAS score

\section{INTRODUCTION}

Distal tibial fractures remain one of the most substantial therapeutic challenges that confront the orthopaedic traumatologist. Incidence of distal tibia fracture is about 9.1 per 100000 per year which includes intra and extraarticular fractures of distal tibia. ${ }^{1}$ Considering that most of distal tibia fractures involve the malleoli, extraarticular distal tibia fractures are relatively less common. Mainly because of its subcutaneous location with precarious blood supply and proximity to the ankle joint most of these fractures are managed with an operative intervention. ${ }^{2,3}$ Closed reduction and intra-medullary interlocking nailing, open reduction and internal fixation (ORIF) with plating, closed reduction and per cutaneous plating, external fixator's are the surgical options with each of these techniques having their own merits and demerits. ${ }^{2,3}$ 
Extra-articular distal tibial fractures are one of the most complicated ones to treat, specially comminuted fractures. It's subcutaneous nature and lack of adequate musculature makes it more prone for soft tissue damage and bone loss. The key to handle these troubling fractures is to skillfully preserve and reconstruct the soft tissues, early mobilisation and functional use of the extremity with the maintenance of satisfactory length and alignment of the fracture. Other factors contributing to complications in management of fractures of distal tibia is tendency for re-displacement of fragments when swelling subside, particularly in comminuted, oblique and spiral fractures and sometimes functional disability if alignment or rotational position of the fragments is imperfect. Sarmiento et al, demonstrated that treatment by closed reduction and cast immobilisation could prevent serious complications of open reduction and internal fixation. His method of functional bracing in the form of patellar tendon bearing cast is the method of cast treatment used most popularly for tibial fractures, but surgeons come across difficulties of mal-alignment, shortening wound management inside the plaster cast, this method is restricted only to low energy tibial fractures. ${ }^{4}$ external fixation devices became the method of treatment of treatment of open tibial fractures in 1980's . These were the main stay of stabilisation of these fractures as these also facilitated the soft tissue care and wound dressing. These devices had certain disadvantages in the form of pin tract infections, longer union time, poor patient compliance and increased complications like infection, may union and non-union. ${ }^{5}$

The disadvantages seen with external fixator's were high and led to the usage of internal fixation devices for stabilisation of tibial fractures as intramedullary nail, plates and screws. Evidence suggests that both intramedullary (IM) nailing and plating are appropriate treatments as IM nailing shows lower rate of delayed wound healing and superficial infection and plating may avoid malunion and knee pain. ${ }^{7}$ IMIL nailing has been reported with higher rate of mal-union because it is difficult to achieve two distally locking screws. ${ }^{6,7}$ newer methods such as minimally invasive approaches combined with biologically friendly internal fixation have become accepted methods of complex fracture treatment. The orthopaedic literature has demonstrated advantages when comparing locking plate techniques with traditional compression plating techniques. The advantages of locking compression plates (LCP) apply most directly to cases of highly communitted fractures, unstable metadiaphyseal segments and osteoporotic fractures. Compression plating requires absolute stability for bone healing. In contrast locking compression plates function as "internal fixators" with multiple anchor points. This type of fixed - angle device converts axial loads across the bone to compressive forces across fracture sites, minimizing gap, length and strain. The surgical treatment of fractures has evolved a great deal since the development of the original "open reduction and internal fixation" technique by the AO group. A mechanically stable fracture -bridging osteosynthesis can be obtained without significant dissection and surgical trauma to the bone and surrounding soft tissues by minimally invasive percutaneous plate osteosynthesis (MIPPO). This technique utilizes indirect reduction and internal fixation with locking compression plates. The objective of biological fixation is to assist physiological process of bone healing wisely and optimally with minimal amount of operative intervention; thus, the emphasis should be laid on maintaining a precarious balance between de-vascularization and mechanical perfection. Objective of our study is to evaluate the clinical and functional outcome of extraarticular distal tibia fracture using distal tibia anatomical LCP using MIPPO technique and to evaluate the post-operative complications in tibia fractures fixed using above technique.

\section{METHODS}

This prospective longitudinal study involves 30 patients with distal tibia fracture, who are admitted in Vydehi Institute of Medical Sciences and Research Centre between January 2017 to 2019. Patients with extraarticular distal tibia fractures in a skeletally matured bone who are medically fit for surgery are included. Patients with open physis, pathological fractures, associated vascular injuries, cognitive disorders and patients who are medically unfit for surgery are excluded. Patients with intrarticular fractures are also excuded. Patients fitting inclusion criteria are admitted after initial management in casualty. Radiological examination pre-operatively are done with $\mathrm{X}$-rays of tibia full length with ankle and knee- anteroposterior (AP) view and lateral view. CT scan was done to delineate fracture pattern. Before subjecting the patients for investigations and surgical procedures written, informed consent was obtained from each patient. All patients were operated in supine position under tourniquet on a radiolucent table. Preoperative antibiotics was given before inflating tourniquet. By manual traction and manipulation reduction was attempted and provisional reduction was confirmed by $\mathrm{C}$-arm.

In MIPPO technique, $1-2 \mathrm{~cm}$ incision was made from the tip of medial malleolus extending proximally to create easy passage, once the medial malleolus is exposed, care taken to protect the saphenous vein. Percutaneous elevators were then be inserted to create a sub muscular, extra periosteal tunnel for the plate. The passage of the plate till sufficiently above fracture site was confirmed with c-arm imaging and second incision was made proximally at the estimated proximal edge of the plate. The plate was then fixed on the tibial surface with Kirschner wire inserted through a fixation bolt. adequate positioning was then confirmed with anteroposterior and lateral imaging. Proximal position of the plate was then checked to ensure central placement of the tibial shaft using c-arm guidance. It was followed by insertion of fixation screws. All the non locking screws was inserted first and after attaining adequate reduction, locking screws was inserted. The AO principles of fixation using LCP was adhered to at every stage of fixation. Wound was closed in layers after 
wash. Post-operative X-rays of tibia full length with ankle and knee- AP view and lateral view was taken. Ankle movements and non weight bearing mobilization was started from $2^{\text {nd }}$ post-operative day according to tolerance level of the patient or associated injuries. I.V. antibiotics was administered for the 3 days post operatively followed by oral antibiotics for another 5 days. Progressive weight bearing was allowed according after 6 weeks. Regular follow up of the patient in OPD with X-rays and functional outcome evaluated as per ankle-hind foot scale (100 points) was done at 3 weeks, 6 weeks, 3 months, 6 months, 1 year and 2 year. All long-term complications like nonunion, malunion, angular deformity, implant breakage, shortening or infection was be recorded if present.

\section{Statistical analysis}

The data thus obtained was compiled using microsoft excel sheet and transferred and analyzed using graph pad prism version 8 . Descriptive statistics was used summarize the data. The data was analyzed using proportion test, Chisquare test. Microsoft word and excel were used to generate graphs, tables etc.

\section{RESULTS}

Total of 30 patients were evaluated in the study. The mean age was 38.5 (range 22-58) years with 22 males (73.3\%) and 8 females (26.6) (Table 1). Right side was injured in $70 \%$ patients (Table 2). Only extraarticular distal tibia fractures are included with $40 \%$ belonging to A1 and $36.7 \%$ belonging to A3 (Table 3). Majority (53.3) of the patients had road traffic accident, $26.7 \%$ of patients fell from height (Table 4).

80 percent of surgeries took less than 60 minutes; 7 percent took 60-90 minutes and 13 percent took 90-120 minutes.

26 patients had concurrent fibula fractures and all fibula fractures were fixed. Among 26, 20 patients were fixed with rush nail after closed reduction whereas 6 patients were fixed with plating. 18 cases were operated within 3 days of injury, 8 cases were operated in 4-10 days, 4 cases were operated in 11-14 days.

Minimum follow up required for inclusion was set at 6 months but more $50 \%$ of our patients were available for follow up of more than 12 months. Mean follow up was $16 \pm 2.4$ months.

16 cases took 3-4 months, 8 cases took 4-5 months, 4 cases took 5-6 months for bony union with mean time for radiological union being 4.6 months.

3 cases had a superficial infection which settled with regular dressing and higher antibiotic, 3 cases had varus angulation of less than 5 degrees in anteroposterior X-ray of ankle, only 1 patient had ankle stiffness. But one of common complication was discoluration of skin which was seen in 16 patients (Table 5).
As per AOFAS ankle hind foot scale, at the end of 1 year, 21 cases $(70 \%)$ of the cases had excellent results, 5 cases had good results, 3 cases had fair results and only 1 case had poor results (Table 6).
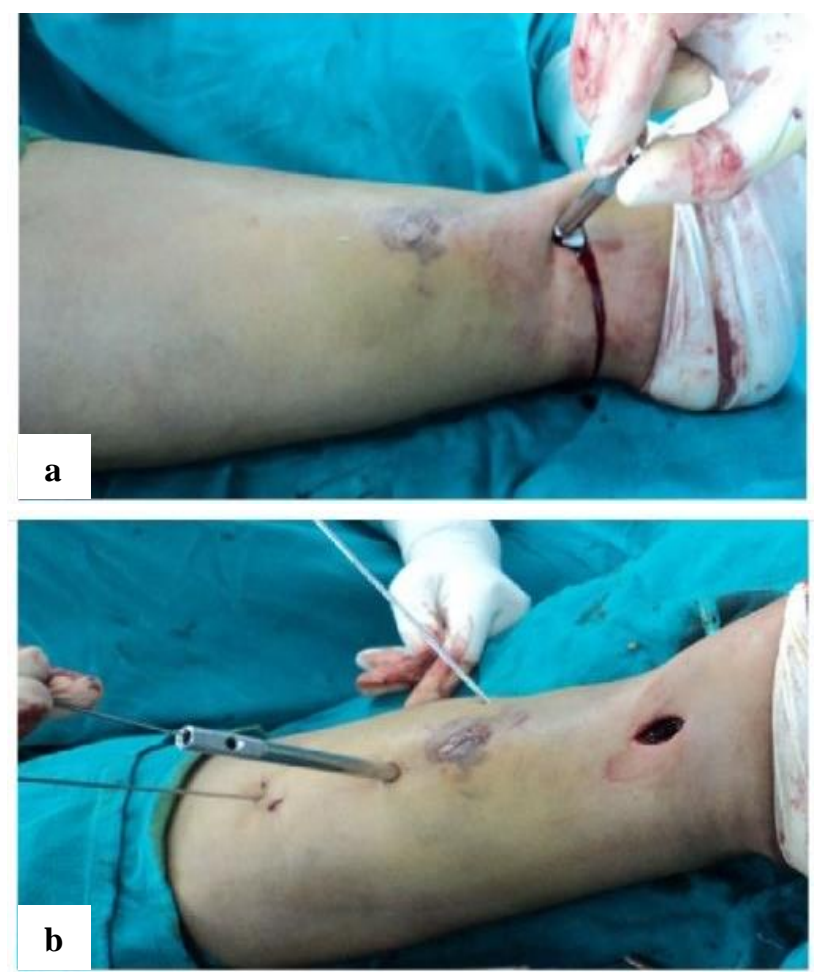

Figure 1: MIPPO technique used for fixation of distal tibia fractures. (a) Plate with sleeve inserted through distal incision, (b) proximal screws inserted with stab incisions.

Table 1: The distribution of cases according to sex.

\begin{tabular}{|lll|}
\hline Sex & Number of cases & Percentage \\
\hline Male & 22 & 73.3 \\
\hline Female & 8 & 26.7 \\
\hline Total & 30 & 100 \\
\hline
\end{tabular}

Table 2: Distribution of cases according to side of fracture.

\begin{tabular}{|lll|}
\hline $\begin{array}{l}\text { Side of } \\
\text { fracture }\end{array}$ & Number of cases & Percentage \\
\hline Right & 21 & 70 \\
\hline Left & 9 & 30 \\
\hline Total & 30 & 100 \\
\hline
\end{tabular}

Table 3: AO/OTA type.

\begin{tabular}{|lll|}
\hline Type & Number of cases & Percentage \\
\hline 43-A1 & 12 & 40 \\
\hline 43-A2 & 7 & 23.3 \\
\hline 43-A3 & 11 & 36.7 \\
\hline Total & 30 & 100 \\
\hline
\end{tabular}


Table 4: Distribution of cases according to mode of injury.

\begin{tabular}{|lll|}
\hline Mode of injury & $\begin{array}{l}\text { Number of } \\
\text { cases }\end{array}$ & Percentage \\
\hline RTA & 16 & 53.33 \\
\hline Fall from height & 8 & 26.77 \\
\hline others & 6 & 20 \\
\hline Total & 30 & 100 \\
\hline
\end{tabular}

Table 5: Complications in our study.

\begin{tabular}{|lll|}
\hline Complications & $\begin{array}{l}\text { Number of } \\
\text { cases }\end{array}$ & Percentage \\
\hline $\begin{array}{l}\text { Superficial wound } \\
\text { infection }\end{array}$ & 3 & 10 \\
\hline Skin discoluration & 16 & 53.3 \\
\hline Ankle stiffness & 1 & 3.33 \\
\hline Varus angulation & 2 & 6.67 \\
\hline
\end{tabular}

Table 6: Clinical results.

\begin{tabular}{|llll|}
\hline $\begin{array}{l}\text { Clinical } \\
\text { results }\end{array}$ & $\begin{array}{l}\text { Ankle-hind } \\
\text { foot scale }\end{array}$ & $\begin{array}{l}\text { Number } \\
\text { of cases }\end{array}$ & Percentage \\
\hline Excellent & $>85$ points & 21 & 70.0 \\
\hline Good & $70-80$ points & 5 & 16.7 \\
\hline Fair & $55-65$ points & 3 & 10.0 \\
\hline Poor & $<50$ points & 1 & 3.3 \\
\hline Total & & 30 & 100 \\
\hline
\end{tabular}

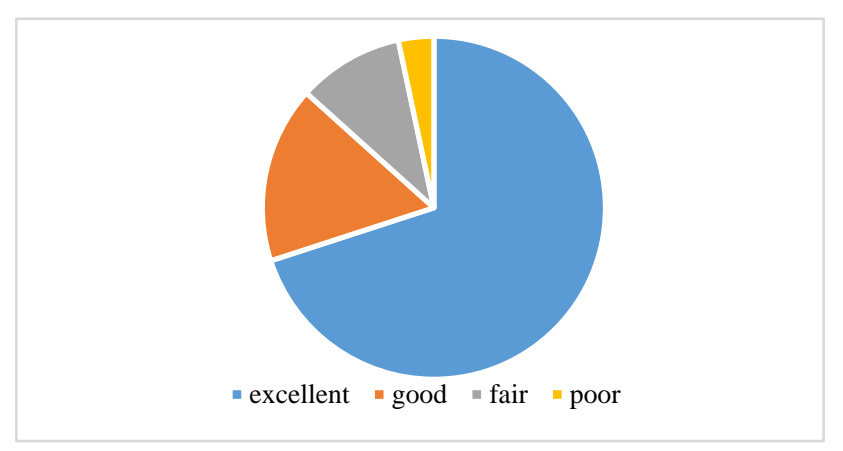

Figure 2: AOFAS score.

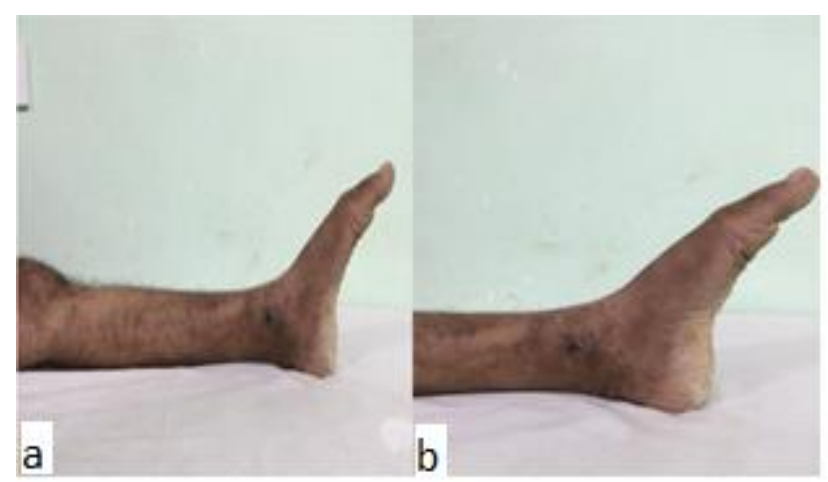

Figure 3 (a and b): Plantar flexion.

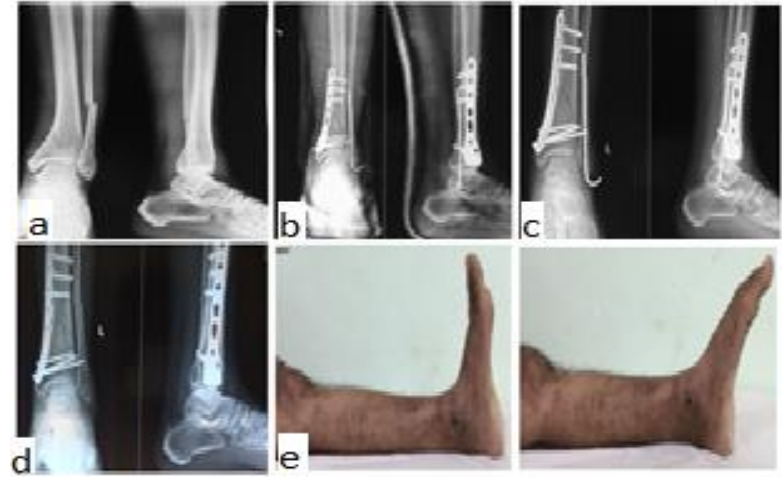

Figure 4: 32 years old with fracture distal tibia-fibula fixed with MIPPO technique, serial follow up X-ray and clinical picture included. (a) Preoperative X-ray,

(b) immediate postoperative, (c) 6 weeks follow up,

(d) 6 months follow up, (e) clinical picture at 6 months follow up.
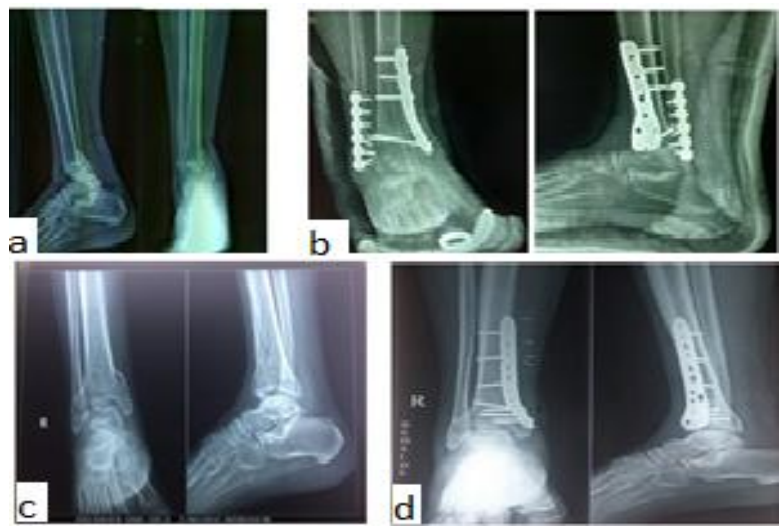

Figure 5: 28 years old distal tibia-fibula fixed with pre-contoured distal tibia plate and reconstruction plate for fibula, (a) preoperative X-ray, (b) follow up at 6 months, 44 years old with distal tibia fracture only fixed using MIPPO technique, (c) preoperative $\mathrm{X}$-ray, (d) immediate postoperative $\mathrm{X}$-ray.

\section{DISCUSSION}

MIPPO technique with pre-contoured LCP used for fixing distal tibia fractures use indirect reduction techniques, and subcutaneous tunnels to pass the plates. Small stab incisions are put for application of locking screws proximally. This prevents iatrogenic injury to vascular supply of the bone and preserve extraosseous blood supply. ${ }^{8}$ Osteogenic fracture hematoma is also preserved, thus gives us a biologically friendly stable fixation which leads early callus formation and bone healing. MIPPO with LCP for distal $3^{\text {rd }}$ tibial fracture has been found to be an effective treatment option. ${ }^{9-11}$

In our study the male preponderance for distal third tibial fractures were high, $73.3 \%$. Similar distribution was seen in studies conducted by Sharma et al. ${ }^{12,13}$ Though age of patients varied (range 22-58 years), most patients were young adults with mean age of 38.5 years. 
Trivial low energy falls usually do not cause distal tibial fractures especially in young patients. In our study, almost half of patients $(53.3 \%)$ had RTA, $26.7 \%$ of patients had fall from height. This is attributable to the fact that most patients belonged to labourers, industrial, construction workers and delivery boys. Distribution in our study is similar to Shrestha et al and Reddy et al who had predominantly RTA as mode of injury in these fractures. ${ }^{14,15}$

We strictly restricted our inclusion criteria to extraarticular distal tibia fractures as the dynamics of intrarticular fractures vary significantly from extraarticular fractures. We had $40 \%$ of our patients belonging to AO type 43.A-1 and $37.7 \%$ AO type 43.A-3; AO type B and C are excluded from our study. Reddy et al had more $60 \%$ type A1 fractures whereas in our studies there were equal number of A1 and A-3 fractures. ${ }^{15}$ Communitted extra-articular distal tibial fractures belonging to type A3 are particularly difficult to achieve proper reduction. we used traction manual traction for achieving alignment and length and used k-wire as joystick in distal fragment to control reduction in sagittal place.

In our study, 26 out of patients had ipsilateral fibular fractures and most of fractures were at the level of tibia. We fixed fibular fractures first before going for accurate reduction of tibial fractures. In most cases, once the fibula was fixed, fractures of tibia fell back into its place requiring only minor manipulation to achieve accurate reduction. In 20 out 26 cases, we achieved closed reduction of fibula and fixed it with rush nail whereas other 6 cases required open reduction with plate fixation.

In our study, most (18 patients) of fractures were fixed within 3 days, even in this 16 out of 18 were operated within $24 \mathrm{hrs}$ of injury. Other cases were delayed by a week if the soft tissue swelling is extensive. Patients who were operated after 10 days were patients who presented late. Our findings regarding union were similar to Cresta et al who noticed that union of fracture site was not affected by delay in surgery. ${ }^{14}$ We recommend that it's better to operate these fractures within 24 hours, if its delayed more than this it's better that we wait till the soft tissue swelling decreases for better soft tissue healing.

Radiological union was achieved in most cases within 5 months with mean time for radiological union was 4.6 months, Lau et al reported 18.7 weeks, Ronga et al reported average radiological union of 22.3 weeks. ${ }^{7,17}$

In our study, common complications that we saw in these patients were skin dis colouration which was present in most patients but marked $53.3 \%$ of our patients. This is particularly significant in patients who needs better cosmetic outcome, this discoluration faded in most patients with time signifying adequate revascularisation and reepithelialisation. About $10 \%$ of our patients had superficial infection which got settled with higher antibiotics and regular dressings. We started ankle mobilisation exercises immediately following surgery attributing ankle stiffness in only one of patient. Singh et al reported 3 cases $(10 \%)$ of malunion. ${ }^{18}$ We had 2 cases with varus malalignment of less than 5 degrees.

In our study we have used AOFAS Ankle-hindfoot scale which is quiet comprehensive modality to evaluate the functional outcome of any foot and ankle pathologies. ${ }^{18}$ Reddy et al used Olerud-Molander ankle score and achieved excellent results in $53.3 \%$ of patients. ${ }^{15} \mathrm{We}$ achieved excellent results in nearly $70 \%$ of our patients with AOFAS score of greater than 85. One patient had poor outcome which was due to delayed union and non complaince of the physiotherapy advise given. Though we achieved union after injecting bone marrow aspirate after 6 months, patient continued to have poor scores.

So, based on the findings of our study, it is found that MIPPO is an excellent technique in achieving fracture reduction and achieve radiological union especially communitted fractures with soft tissue injury. MIPPO technique decrease operating time, blood loss and helps us to minimise soft tissue injury in distal tibia fractures which only minimal subcutaneous soft tissue cover. Few complications seen like superficial infection, skin discoluration were easily managed. With proper operative technique and physiotherapy, complications like ankle stiffness and malalignment can completely be avoided. Thus, MIPPO technique helped us achieve an excellent functional outcome in majority of patients. Limitation of study is that it has a small sample size, follow up of only 1-year.

\section{CONCLUSION}

With the use of pre-contoured anatomical locking plates used, MIPPO technique is effective in extra articular distal tibia fractures where it not only helps in decreasing operating time but also in achieving excellent functional outcome with radiological union the fractures by preserving osseous vascularity and minimal soft tissue insult due to surgery. MIPPO is safer and efficacious modality of the treatment for extra-articular distal tibial fractures.

Funding: No funding sources Conflict of interest: None declared

Ethical approval: The study was approved by the institutional ethics committee

\section{REFERENCES}

1. Bergdahl DWC. Epidemiology and incidence of tibia fractures in the Swedish Fracture Register. Injury. 2018;49(11):2068-74.

2. White TO, Guy P, Cooke CJ, Kennedy SA, Droll KP, Blachut PA, et al. The results of early primary open reduction and internal fixation for treatment of OTA 43.C-type tibial pilon fractures: a cohort study. J Orthop Trauma. 2010;24(12):757-63. 
3. Zhao L, Li Y, Chen A, Zhang Z, Xi J, Yang D. Treatment of type $\mathrm{C}$ pilon fractures by external fixator combined with limited open reduction and absorbable internal fixation. Foot Ankle Int. 2013;34(4):534-42.

4. Austin RT. The Leicester Royal Infirmary UK. The Sarmiento tibial plaster: a prospective study of 145 fractures. Injury. 1981;13(1):10-22.

5. Justin E, Richards MD, Magill M, Marc A, Tressler DO, Shuler FD, et al. Southeast Fracture Consortium. External Fixation Versus ORIF for Distal Intraarticular Tibia Fractures. Orthopedics. 2012;35(6):862-7.

6. Kneifel T, Buckley R. A comparison of one versus two distal locking screws in tibial fractures treated with unreamed tibial nails: a prospective randomized clinical trial. Injury. 1996;27:271-3.

7. Mao Z, Wang G, Zhang L. Intramedullary nailing versus plating for distal tibia fractures without articular involvement: a meta-analysis. J Orthop Surg Res. 2015;10:95.

8. Teitz CC, Carter DR, Frankel VH. Problems associated with tibial fractures with intact fibulae. J Bone Joint Surg Am. 1980;62:770-6.

9. Ronga M, Longo UG, Maffulli N. Minimally Invasive Locked Plating of Distal Tibia Fractures is Safe and Effective. Clinical Orthop Rel Res. 2010;68(4):975-82.

10. Bahari S, Leneham B, Khan H, Mcelwain JP. Minimally invasive percutaneous plate fixation of distal tibia fractures. Acta Orthop Belg. 2007;73:63540.

11. Gupta RK, Rohilli RK, Sangwan K, Singh V, Walia S. Locking plate fixation in distal metaphyseal tibial fractures: series of 79 patients. Inter Ortho. 2010;34:1285-90.
12. Collinge C, Protzman R. Outcomes of minimally invasive plate osteosynthesis for metaphyseal distal tibia fractures. J Ortho Trauma. 2010;24:24-29.

13. Sharma CB, Waikhom S. Management of Fracture Distal Tibia with locking ledial Metaphyseal plate. J Evidence Based Med Healthcare. 2015;2(29):420942.

14. Shrestha D, Acharya BM, Shrestha FPM. Minimally invasive plate Osteosynthesis with locking compression plate for distal diametaphyseal tibia fracture. Kathmandu Univ Med J. 2011;(34):62-8.

15. Reddy M, Kumar AV, Shaik T. Outcome of distal tibia fractures managed with locking compression plate using MIPPO technique. IJOS. 2019;5(3):30410.

16. Abdulla S, Senna A. Minimally Invasive Plate Osteosynthesis for Distal Tibial Fractures. J American Sci. 2013;9(10).

17. Shikhar D, Singh, Manohar PV, Butala R. Mnimally invasive plate osteosynthesis in management of distal tibial fractures. IJSR. 2015;4(6).

18. American Orthopaedic Foot and Ankle Society (AOFAS) Ankle-Hindfoot Score: a study protocol for the translation and validation of the Dutch language version. American Orthopaedic Foot and Ankle Society (AOFAS) Ankle-Hindfoot Score: a study protocol for the translation and validation of the dutch language version. BMJ Open. 2017;7(2):012884.

Cite this article as: Mandri A, Jagadeesh N, Nanjareddy S, Shivalingappa VM. The prospective analysis of functional outcome of osteosynthesis of extra-articular distal tibia fractures using minimally invasive percutaneous plate osteosynthesis technique. Int J Res Orthop 2020;6:536-41. 